\title{
GGUMLINK: A computer program to link parameter estimates of the generalized graded unfolding model from item response theory
}

\author{
JAMES S. ROBERTS and CHUN-WEI HUANG \\ University of Maryland, College Park, Maryland
}

\begin{abstract}
The generalized graded unfolding model (GGUM) is an item response theory (IRT) model that implements symmetric, nonmonotonic, single-peaked item characteristic curves. The GGUM is appropriate for measuring individual differences for a variety of psychological constructs, especially attitudes. Like other IRT models, the location and scale (i.e., the metric) of parameter estimates from the GGUM are data dependent. Therefore, parameter estimates from alternative calibrations will generally not be comparable, even when responses to the same items are analyzed. GGUMLINK is a computer program developed to reexpress parameter estimates from two separate GGUM calibrations in a common metric. In this way, the results from separate calibrations of model parameters can be compared. GGUMLINK can secure a common metric by using one of five methods that have recently been generalized to the GGUM. The GGUMLINK executable program is available free and may be downloaded from http://www.education.umd.edu/EDMS/tutorials/index.html.
\end{abstract}

The generalized graded unfolding model (GGUM) is a unidimensional item response theory (IRT) model for polytomous responses developed by Roberts, Donoghue, and Laughlin (2000). The model implements symmetric, nonmonotonic, single-peaked response functions in which the probability that a respondent will achieve a higher item score is a function of the distance between the locations of the individual and the item on an underlying latent continuum. Specifically, a higher item score is expected to the extent that the individual is located closer to an item. The model is consistent with the notion of a proximity-based response process (Coombs, 1964), and it is appropriate for measuring a variety of psychological constructs, such as attitudes, preferences, and individual locations in certain developmental processes that occur in stages. Being an IRT model, the GGUM simultaneously measures individual differences and scales the characteristics of items, using responses from a single sample.

Roberts et al. (2000) defined the category probability function for the GGUM as the equation located at the top of the next page, where $P\left(Z_{i}=z \mid \theta_{j}\right)$ is the probability that an individual located at $\theta_{j}$ on the latent continuum will respond to item $i$, using response category $z ; z=0,1, \ldots$, $C$; $C$ is equal to the number of item response categories

This material is based on work supported by the National Science Foundation under Grant 0133019, awarded by the Methodology, Measurement, and Statistics program in the Division of Social and Economic Sciences. Correspondence should be sent to J. S. Roberts, Department of Measurement, Statistics, and Evaluation, 1230F Benjamin Building, University of Maryland, College Park, MD 20742 (e-mail: jr245@umail.umd.edu). minus $1 ; \delta_{i}$ is the location of item $i$ on the latent continuum; $\alpha_{i}$ is the discrimination parameter for item $i ; \tau_{i k}$ is one of $k$ "subjective" response category thresholds for item $i$, with $k=0,1, \ldots, C$; and $M=2 C+1$.

This category probability function is plotted in Figure 1 for a hypothetical attitude questionnaire item with four response categories (e.g., strongly disagree, disagree, agree, and strongly agree). The item parameters for this hypothetical item are $\delta_{i}=0.5, \alpha_{i}=1.1, \tau_{i 0}=0$, $\tau_{i 1}=-2.0, \tau_{i 2}=-1.2$, and $\tau_{i 3}=-0.75$. The GGUM provides the probability that an individual located at $\theta_{j}$ on the latent (attitude) continuum will use a given item response. Each probability curve corresponds to a different response, and at any location on the $\theta$ axis, these four probability curves sum to 1 . With this hypothetical item, the relative probabilities of responses indicative of successively higher levels of agreement increase as $\left|\theta_{j}-\delta_{i}\right|$ decreases. Figure 2 illustrates the expected value of $Z_{i}$ as a function of $\theta_{j}$ for the same hypothetical item. In this case, $Z_{i}=0,1,2$, and 3 , where 0 corresponds to strongly disagree and 3 corresponds to strongly agree. As is shown in Figure 2, the expected level of agreement increases as $\left|\theta_{j}-\delta_{i}\right|$ approaches zero. This feature is the hallmark of any unfolding item response model.

The model in Equation 1 can be constrained in a variety of ways. For example, the subjective response category thresholds may be forced to remain constant across all items, yielding one set of $C$ thresholds. Alternatively, the thresholds for item $i$ may be constrained to be equally distant from each other on the latent continuum, and this interthreshold distance can be identified with a single parameter, $\lambda_{i}$. The interthreshold distance parameter can also be forced to be equal across all items, which yields 


$$
P\left(Z_{i}=z \mid \theta_{j}\right)=\frac{\exp \left\{\alpha_{i}\left[z\left(\theta_{j}-\delta_{i}\right)-\sum_{k=0}^{z} \tau_{i k}\right]\right\}+\exp \left\{\alpha_{i}\left[(M-z)\left(\theta_{j}-\delta_{i}\right)-\sum_{k=0}^{z} \tau_{i k}\right]\right\}}{\sum_{w=0}^{C}\left(\exp \left\{\alpha_{i}\left[w\left(\theta_{j}-\delta_{i}\right)-\sum_{k=0}^{w} \tau_{i k}\right]\right\}+\exp \left\{\alpha_{i}\left[(M-w)\left(\theta_{j}-\delta_{i}\right)-\sum_{k=0}^{w} \tau_{i k}\right]\right\}\right)}
$$

a single $\lambda$ parameter. There are, thus, four alternative versions of the GGUM that one can obtain by placing different constraints on the nature of the subjective response category thresholds. In addition, one can allow the discrimination parameter to vary across items or constrain it to be constant across items. These two options for item discrimination parameters can be crossed with the four models produced from alternative threshold formulations to produce a total of eight possible models in the GGUM family.

Roberts (2001b) released a free computer program, called GGUM2000, to estimate model parameters for any of the eight possible models in the GGUM family. The program implements a marginal maximum likeli- hood (MML) solution for item parameters and an expected a posteriori (EAP) solution for person parameters. Each of these solutions utilizes a prior distribution for $\theta_{j}$, which is defined by GGUM2000 to be normal with a mean of 0 and a standard deviation of 1 . Simulation studies suggest that solutions such as those implemented by GGUM2000 are robust to this choice of a prior distribution (Bock \& Aitkin, 1981; Roberts, 2000; Roberts, Donoghue, \& Laughlin, 2002; Seong, 1990). For example, Roberts et al. (2002) found that accurate MML estimates of item parameters could be obtained using an $N(0,1)$ prior when the true distribution of $\theta_{j}$ was bimodal, positively skewed, or negatively skewed and the sample size was 1,000 or greater. Similarly, accurate

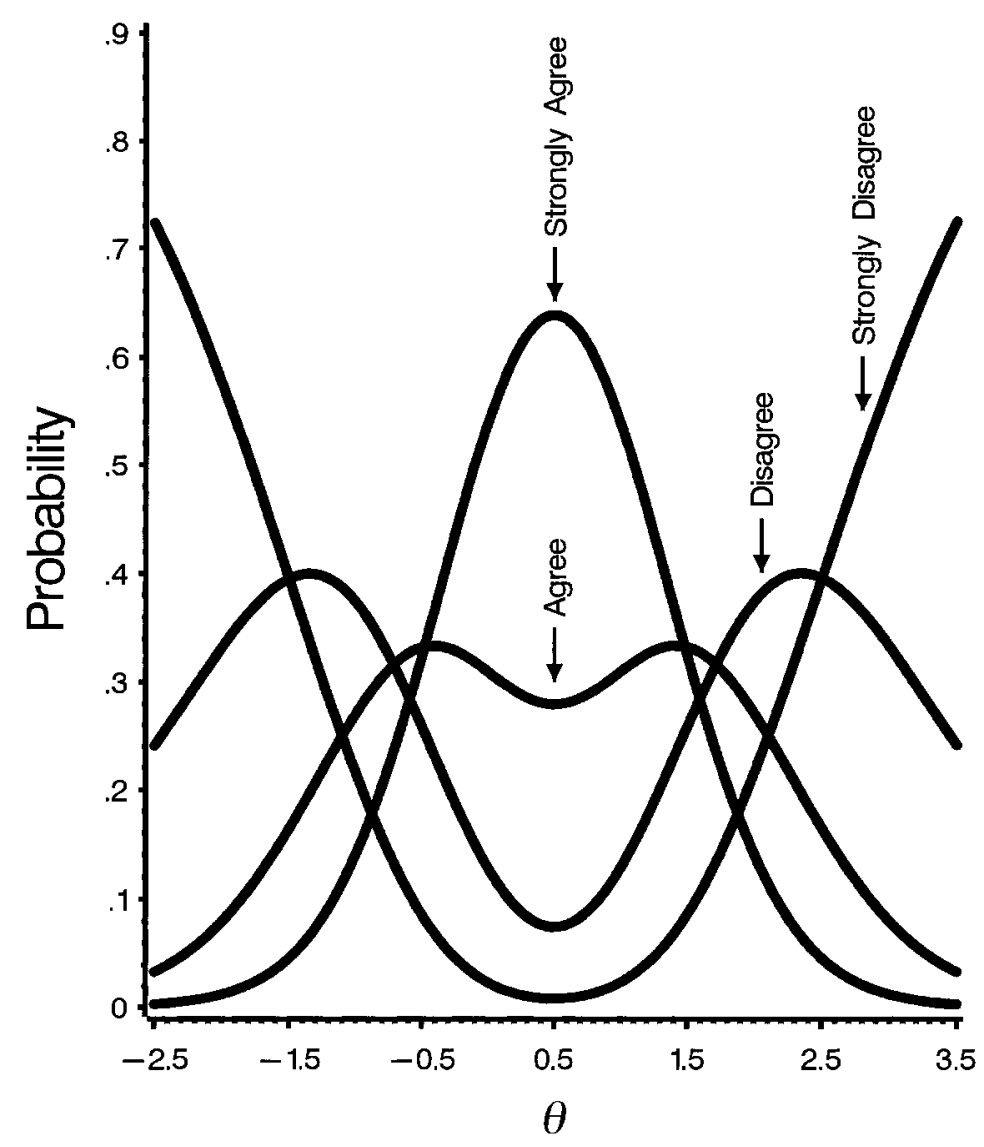

Figure 1. Category probability functions for a hypothetical attitude item under the GGUM. The item has four response categories (strongly disagree, disagree, agree, and strongly agree). Each curve yields the conditional probability of a particular response, given a value for $\theta$, where $\theta$ is a respondent's location on the latent continuum. 


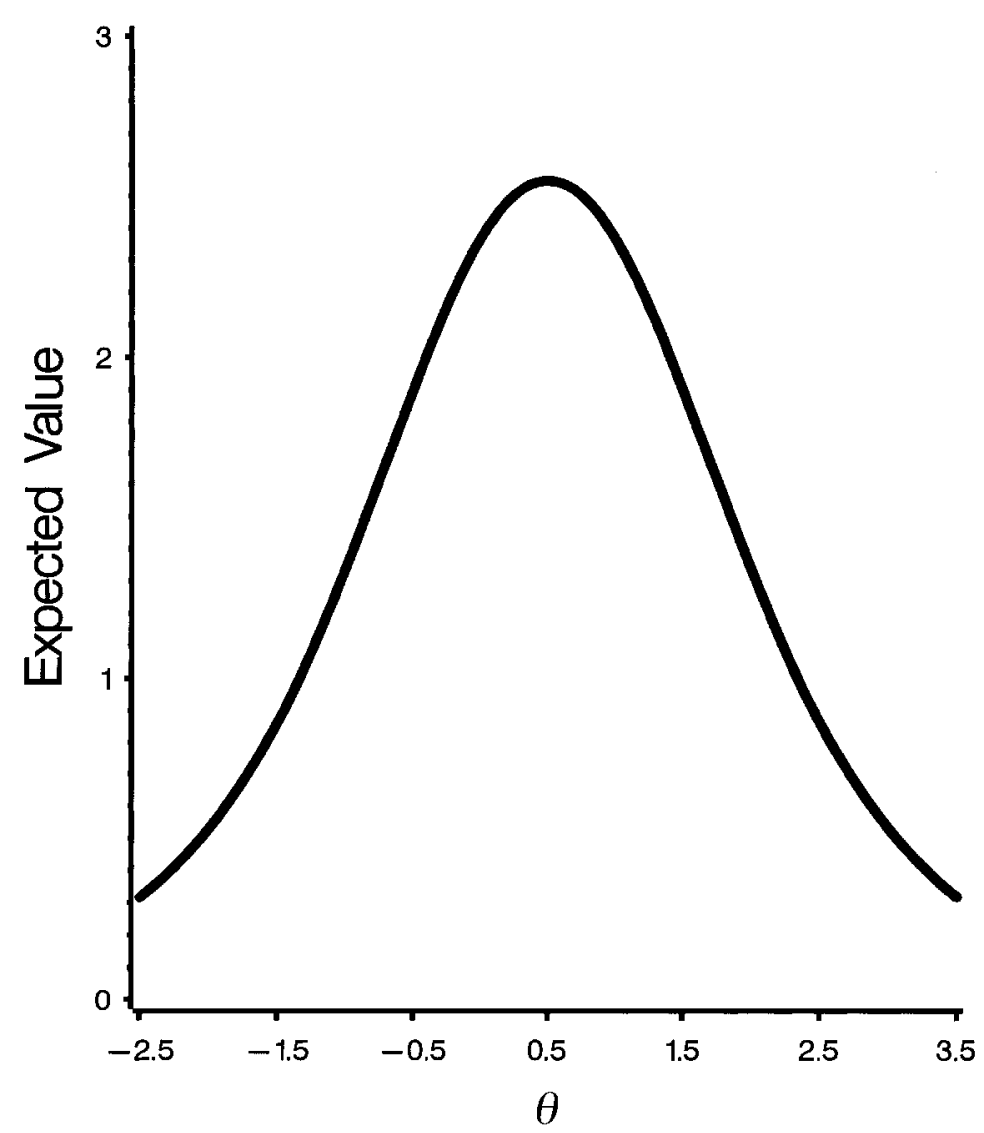

Figure 2. The expected value function for the same hypothetical item under the GGUM. Responses are coded as $0=$ strongly disagree, $1=$ disagree, $2=$ agree , $\mathbf{3}=$ strongly agree , and then the expected value of these codes is taken.

EAP estimates of $\theta_{j}$ could generally be obtained when estimates were based on 20 or more uniformly spaced items. Such findings are due to the fact that the MML and EAP procedures are Bayesian estimation procedures and, thus, the resulting estimates are influenced by the prior distribution only to the extent that the data are uninformative. When MML estimates of item parameters are calibrated using a large sample of informative item responses, differences between the prior distribution of $\theta_{j}$ and its true distribution will have very little impact on the resulting estimates. Similarly, differences between the prior and the true distributions of $\theta_{j}$ will have little effect on the EAP estimate of $\theta_{j}$ when a suitable number of informative item responses is available.

IRT models provide several benefits when they fit the data at hand. Specifically, they provide estimates of precision for each model parameter, which enables one to determine how well each respondent has been measured (i.e., determine the standard error of each $\theta_{j}$ estimate). Parametric IRT models also provide item-invariant interpretations of $\theta_{j}$, which allow one to estimate $\theta_{j}$ for different individuals, using different items. This, in turn, allows for tailored tests and computerized adaptive testing.
Finally, these models provide sample-invariant interpretations of item parameters, which enables one to develop large item pools and provide alternative means to equate test scores and assess differential item functioning. The latter two features implicitly assume that all item and person parameters in a given application are expressed on a common metric. Like other multiparameter IRT models, the metric (i.e., the location and scale) of the parameter estimates derived from the GGUM cannot be uniquely determined from the likelihood function alone. However, the mean and standard deviation of the prior distribution used to estimate model parameters constrains the location and scale of the estimates to unique, yet arbitrary, values. This yields a metric for model parameter estimates that is dependent on the specific data used to calibrate the estimates. Consequently, a means by which to transform parameter estimates to a common metric is necessary to obtain the invariance properties of parametric IRT models.

Consider an attitude measurement situation in which the GGUM is used to investigate attitudes toward capital punishment. Suppose that model parameter estimates associated with a single attitude questionnaire are calcu- 
lated twice using two independent samples of respondents. This strategy is often used to examine whether questionnaire items function similarly in two demographic groups. The MML and EAP estimation algorithms implemented in the GGUM2000 software will scale parameter estimates under the assumption that true attitudes are distributed with a mean of 0 and a variance of 1 . If the means and variances of true attitudes differ in the two samples, the metric of the parameter estimates obtained from the two calibrations will also differ. Consequently, the resulting estimates must be transformed to achieve a common metric before they can be compared across samples.

What is needed is a mechanism by which to transform the parameter estimates from the two separate calibrations to a common metric so that parameter estimates can be compared. This process will be referred to as linking parameter estimates. Roberts (2001a) has generalized several strategies for linking item parameters from traditional IRT models to the GGUM family. We will briefly review these strategies below and then describe a new computer program called GGUMLINK that implements each strategy.

\section{Alternative Strategies to Link GGUM Parameters}

Mean-sigma method based on item locations. Suppose that responses from two groups of individuals are calibrated separately using GGUM2000 software. The responses from the two groups may have been obtained from a single form of an attitude questionnaire or from two distinct forms that contain common items. (In the former case, all the questionnaire items are treated as common items.) Linking constants can be estimated from the means and standard deviations of the item location estimates for common items as follows:

$$
\hat{B}=\overline{\hat{\delta}}_{1}-\frac{s_{\hat{\delta}_{1}}}{s_{\hat{\delta}_{2}}} \overline{\hat{\delta}}_{2},
$$

and

$$
\hat{A}=\frac{s_{\hat{\delta}_{1}}}{s_{\hat{\delta}_{2}}},
$$

where $\overline{\hat{\delta}}_{1}$ and $\overline{\hat{\delta}}_{2}$ are the means of the item location estimates for the common items from the first and the second calibrations, respectively, and $s_{\hat{\delta}_{1}}$ and $s_{\hat{\delta}_{2}}$ are the corresponding standard deviations. $\hat{B}$ is an estimate of the location constant, and $\hat{A}$ is an estimate of the scale constant. Together, these estimates can be used to transform the GGUM person parameter estimates from the metric of the second calibration to that of the first as follows:

$$
\hat{\theta}_{j_{2}, 21}=\hat{A} \hat{\theta}_{j_{2}, 2}+\hat{B}
$$

where $\hat{\theta}_{j_{2}, 21}$ refers to the latent trait (i.e., attitude) estimate for the $j_{2}$ th individual from the second calibration after it has been transformed to the metric of the first calibration. Similarly, item parameters from the second cali- bration can be transformed to the metric of the first as follows:

$$
\begin{gathered}
\hat{\delta}_{i, 21}=\hat{A} \hat{\delta}_{i, 2}+\hat{B}, \\
\hat{\tau}_{i k, 21}=\hat{A} \hat{\tau}_{i k, 2},
\end{gathered}
$$

and

$$
\hat{\alpha}_{i, 21}=\frac{\hat{\alpha}_{i, 2}}{\hat{A}} .
$$

If the interthreshold distances in the GGUM are constrained to be equal for a given item, Equation 6 becomes

$$
\hat{\lambda}_{i, 21}=\hat{A} \hat{\lambda}_{i, 2} \text {. }
$$

Note that the value of the category probability function remains the same when the transformed parameters in Equations 4-7 are substituted into Equation 1 in place of the original parameters from the second calibration. This occurs because the GGUM is invariant to changes in the location and scale of the model parameters.

Mean-sigma method based on item steps. The solution for $\hat{A}$ and $\hat{B}$ given above includes information about item location parameters from the first and the second calibrations, $\hat{\delta}_{i, 1}$ and $\hat{\delta}_{i, 2}$, only. Additional information can be used to estimate linking constants by reparameterizing item and threshold parameters into step parameters (Roberts, 2001a) as follows:

$$
\xi_{i s}=\delta_{i}+\tau_{i s},
$$

where $s=0,1, \ldots, C$. There will be as many estimable $\xi_{i s}$ parameters as there are response categories for the $i$ th item. The estimable step parameters can be used to develop linking constants with the mean-sigma procedure:

$$
\hat{B}=\overline{\hat{\xi}}_{1}-\frac{s_{\hat{\xi}_{1}}}{s_{\hat{\xi}_{2}}} \bar{\xi}_{2},
$$

and

$$
\hat{A}=\frac{s_{\hat{\xi}_{1}}}{s_{\hat{\xi}_{2}}},
$$

where the means and standard deviations are taken across all estimable $\xi_{\text {is }}$ for common items in the first and the second calibrations (denoted simply as $\hat{\xi}_{1}$ and $\hat{\xi}_{2}$, respectively). These estimated linking constants can then be used to transform the metric of the initial parameters from the second calibration as indicated in Equations 4-7. This method uses more information about the GGUM parameter estimates than does the mean-sigma method based solely on item locations. However, item thresholds and, thus, item steps are more difficult to estimate than are item locations (Roberts et al., 2002). Therefore, the additional information provided by the item steps could potentially be outweighed by estimation error in a given situation (Roberts, 2001a).

Mean-mean method based on item locations and item discriminations. Linking constants may also be estimated using the means of both item locations and 
item discriminations for the common items in the alternative calibrations as follows:

$$
\hat{B}=\overline{\hat{\delta}}_{1}-\left(\frac{\overline{\hat{\alpha}}_{2}}{\hat{\hat{\alpha}}_{1}}\right) \overline{\hat{\delta}}_{2},
$$

and

$$
\hat{A}=\frac{\overline{\hat{\alpha}}_{2}}{\hat{\alpha}_{1}} .
$$

In these equations, $\hat{\delta}_{1}$ and $\hat{\delta}_{2}$ refer to estimated item locations of common items in the first and the second calibrations, respectively, whereas $\hat{\alpha}_{1}$ and $\hat{\alpha}_{2}$ are the corresponding estimates of item discrimination parameters. The estimates of linking constants from this method may be advantageous because they are based on both item location and item discrimination parameters and, thus, contain more information about the model parameters than do estimates from the mean-sigma method based solely on item locations. However, parameter recovery simulations suggest that the item discrimination parameters are slightly more difficult to estimate than are the item location parameters (Roberts et al., 2002), and this may outweigh the benefits of including information about the item discrimination parameters in a given situation (Roberts, 2001a). Estimates derived with the mean-mean method may also be superior to those produced with the mean-sigma method, because means are generally more robust to outliers than are standard deviations (Baker \& Al-Karni, 1991).

Mean-mean method based on item steps and item discriminations. The mean-mean method may also be applied using item steps instead of item locations:

$$
\hat{B}=\overline{\hat{\xi}}_{1}-\left(\frac{\overline{\hat{\alpha}}_{2}}{\overline{\hat{\alpha}}_{1}}\right) \overline{\hat{\xi}}_{2} .
$$

The $\hat{A}$ for this method is identical to that in Equation 13 . The potential benefits and pitfalls of this technique are similar to those for the mean-mean method based on item locations and item discriminations. This method uses information about all GGUM item parameters for common items, but the information provided by threshold parameters, and perhaps the discrimination parameters, may be degraded to some extent by estimation error in a given situation.

Item characteristic curve method. The item characteristic curve (ICC) method attempts to alter the metric of one calibration so that the resulting ICCs for common items are as similar as possible to those in another cali- bration. With regard to the GGUM, the ICC for the $i$ th item is simply the conditional expected value of a response to that item, $Z_{i}$, at a given value of $\theta_{j}$ :

$$
E_{i}\left(\theta_{j}\right)=\sum_{z=0}^{C} z\left[P\left(Z_{i}=z \mid \theta_{j}\right)\right] .
$$

In order to describe the logic of the ICC method for estimating linking constants, some definitions must first be given. Let $E_{i, 1}\left(\hat{\theta}_{j, 1}\right)$ denote the ICC for the $i$ th common item in the first calibration group, given the $j_{1}$ th individual's latent trait estimate derived in that calibration. Similarly, let $E_{i, 2}\left(\hat{\boldsymbol{\theta}}_{j_{2}, 2}\right)$ represent the ICC for the $i$ th common item from the second calibration, given the $j_{2}$ th individual's latent trait estimate derived in that calibration. Let $E_{i, 1}\left(\hat{\theta}_{j_{2}, 21}\right)$ denote the ICC for the $i$ th common item from the first calibration, given the $j_{2}$ th individual's transformed latent trait estimate. The transformation takes the $j_{2}$ th individual's trait estimate from the second calibration and transforms it to the metric of the first as defined in Equation 4. In an analogous fashion, $E_{i, 2}\left(\hat{\theta}_{j_{1}, 12}\right)$ refers to the ICC for the $i$ th common item from the second calibration, given the $j_{1}$ th individual's transformed latent trait estimate. This transformation is given by

$$
\hat{\theta}_{j_{1}, 12}=\frac{\hat{\theta}_{j_{1}, 1}-\hat{B}}{\hat{A}} .
$$

Note that this is the inverse of the transformation given in Equation 4. With these definitions in place, the ICC method determines the values of $\hat{A}$ and $\hat{B}$ so that the following quadratic loss function is minimized (see Equation 17 at bottom of page), where $I$ is the number of common items, $J_{1}$ is the number of respondents in the first calibration group, and $J_{2}$ is the number of respondents in the second calibration group. The loss function, $Q$, is composed of two basic functions within the outer parentheses. The function on the left accumulates squared differences between the ICCs for common items in the second calibration and those for corresponding items in the first calibration after they have been reexpressed in the metric defined by Equation 4. The function on the right of Equation 17 operates in the reverse direction. Specifically, it accumulates squared differences between the ICCs for common items in the first calibration and those for corresponding items in the second calibration after transforming the latter ICCs according to Equation 16. Minimization of Equation 17 with respect to $\hat{A}$ and $\hat{B}$ can be performed using alternative numerical methods. In the GGUMLINK program, this minimization is performed using a Newton-Raphson technique.

$$
Q=\sum_{i=1}^{I}\left\{\sum_{j_{2}=1}^{J_{2}}\left[E_{i, 2}\left(\hat{\theta}_{j_{2}, 2}\right)-E_{i, 1}\left(\hat{\theta}_{j_{2}, 21}\right)\right]^{2}+\sum_{j_{1}=1}^{J_{1}}\left[E_{i, 1}\left(\hat{\theta}_{j_{1}, 1}\right)-E_{i, 2}\left(\hat{\theta}_{j_{i}, 12}\right)\right]^{2}\right\}
$$


The ICC method utilizes information about all the item parameters simultaneously. However, the solution is based on discrepancies among ICCs, rather than on actual parameter estimates, and thus, some of the estimation error encountered in one item parameter may be offset by opposing estimation error in another item parameter. Consequently, the estimated ICC for a given item may be accurate even when there is noticeable estimation error in corresponding item parameters (Hulin, Lissak, \& Drasgow, 1982; Linn, Levine, Hastings, \& Wardrop, 1981). Equation 17 accumulates squared differences in both directions, and this leads to a symmetric solution for $\hat{A}$ and $\hat{B}$ that is not dependent on which calibration is chosen to serve as the target metric. In fact, this property is true of all the linking methods discussed in this article.

\section{The GGUMLINK Program}

The GGUMLINK program estimates linking constants, using each of the five methods discussed in the previous section. The user is free to choose which of these estimates will ultimately be used to transform the metric of model parameters from the second calibration. The program is written to complement the GGUM2000 software, and it will automatically read the parameter estimate files produced by GGUM2000. The GGUMLINK program is an executable module developed with Compaq Visual FORTRAN Version 6.6a. It runs under the Windows 95, 98, and 2000 operating systems. A detailed user manual, in PDF format, is included with the program, along with some operational examples.

User input. The GGUMLINK program requires user input in the form of text-based commands. These commands may be entered in an interactive or a batch mode. Upon execution of the program, the user can choose to implement either mode. If an interactive mode is chosen, the program prompts the user for each command. Alternatively, the commands may be entered into an ASCII file for batch processing. An example batch command file is given in the Appendix.

Program output. The GGUMLINK generates five ASCII files as it executes. The first of these files is a generic program output file. It contains information about the application at hand, including estimates of location constants under the five methods, target and transformed item parameter listings using the transformation method chosen by the user, and summary statistics for both target and transformed model parameters. An ex- ample program output file is given in the Appendix. There are four parameter files that are produced in addition to the program output file. Two of these files contain target parameters (i.e., parameters from the first calibration). The first of these files contains item parameters, whereas the second contains person parameters. The remaining two files contain the transformed item parameters and transformed person parameters from the second calibration. The four parameter files created by the GGUMLINK program are formatted for easy input into other software packages to facilitate subsequent analysis.

Program availability. The GGUMLINK program is available free of charge. The program is distributed over the Internet from http://www.education.umd.edu/EDMS/ tutorials/index.html. Instructions for downloading and installing the software are included on the "Free Software" page at that Web site.

\section{REFERENCES}

BAKER, F. B., \& AL-KARNI, A. (1991). A comparison of two procedures for computing IRT equating coefficients. Journal of Educational Measurement, 28, 147-162.

Bock, R. D., \& AitKin, M. (1981). Marginal maximum likelihood estimation of item parameters: Application of an EM algorithm. Psychometrika, 46, 443-459.

Coombs, C. H. (1964). A theory of data. New York: Wiley.

Hulin, C. L., LissaK, R. I., \& Drasgow, F. (1982). Recovery of twoand three-parameter logistic item characteristic curves: A Monte Carlo study. Applied Psychological Measurement, 6, 249-260.

Linn, R. L., Le Vine, M. V., Hastings, C. N., \& WARdrop, J. L. (1981). Item bias in a test of reading comprehension. Applied Psychological Measurement, 5, 159-173.

ROBERTS, J. S. (2000, July). Estimation of parameters in the generalized graded unfolding model using adjusted quadrature. Paper presented at the 65th annual meeting of the Psychometric Society, Vancouver, BC.

RoBERTS, J. S. (2001a, April). Equating parameters of the generalized graded unfolding model. Paper presented at the annual meeting of the American Educational Research Association, Seattle, WA. (Available from the ERIC Clearinghouse on Assessment and Evaluation, Document ED457187.)

RoberTs, J. S. (2001b). GGUM2000: Estimation of parameters in the generalized graded unfolding model [Abstract]. Applied Psychological Measurement, 25, 38.

Roberts, J. S., Donoghue, J. R., \& LAughlin, J. E. (2000). A general item response theory model for unfolding unidimensional polytomous responses. Applied Psychological Measurement, 24, 3-32.

Roberts, J. S., Donoghue, J. R., \& LAughlin, J. E. (2002). Characteristics of MML/EAP parameter estimates in the generalized graded unfolding model. Applied Psychological Measurement, 26, 192-207.

SEONG, T. (1990). Sensitivity of marginal maximum likelihood estimation of item and ability parameters to the characteristics of the prior ability distributions. Applied Psychological Measurement, 14, 299-311.

\section{APPENDIX}

\section{Description of the Example}

The GGUMLINK example shown below is based on simulated responses to 21 items from two independent groups. Item responses were generated on the basis of Equation 1, using five response categories per item. True item parameters were generated in a manner similar to that described by Roberts et al. (2002). The true $\theta$ parameters for simulees in the first group were generated from a normal distribution with a mean of 0 and a standard deviation of 1 . Those for the second group were generated from a normal distribution with a mean of -0.15 and a standard deviation of 1.1. GGUM2000 software was used to estimate model parameters separately in each group. Parameters were estimated under the assumption that the mean and the standard devi- 
APPENDIX (Continued)

ation of true $\theta$ were equal to 0 and 1, respectively. Consequently, the metrics of parameter estimates obtained from the two calibrations were different. The unit of the estimates from the second calibration was too small and required expansion (true $A=1.1$ ), whereas the mean of these estimates was too large and required reduction (true $B=-0.15$ ). The number of simulees in each group was originally 2,000, but GGUM2000 discarded 2 simulees in the first group and 4 simulees in the second group that failed to exhibit a minimal amount of agreement with any of the 21 items. This left 1,998 and 1,996 simulees in the two groups, respectively.

GGUMLINK was used to estimate the linking constants, $A$ and $B$, given the parameter estimates from the GGUM2000 software. Estimates of linking constants were based on all 21 items because item characteristics (i.e., true item parameters) were identical in the two groups. This mimicked the situation in which a common questionnaire is administered to independent samples. Although the GGUMLINK software estimates $A$ and $B$ with all of the methods discussed earlier in this paper, the estimates derived with the ICC method were chosen to transform the model parameters so as to achieve a common metric. This choice was based on preliminary simulation results that suggested that the ICC method yields estimates of linking constants that are generally as accurate as (or slightly more accurate than) those from the other methods and produces fewer outliers (Roberts, 2001a). The ICC method uses an iterative algorithm that requires starting values for $A$ and $B$. The user can supply starting values or let the program supply them. In the latter case, estimates derived from the mean-mean method based on item locations are used as starting values for the ICC method. In this example, starting values were generated by the program.

\section{Example Command File}

The following example command file can be used to link parameter estimates for a common form with 21 items, where each item has five response categories. The commands required from the user are given on the left of each line in uppercase. Explanations of the commands are given on the right of each line in lowercase.

8
C:\GGUMLUSE\FT16M8A
21
C:\GGUMLUSE\FT17M8A
1998
C:\GGUMLUSE\FT16M8B
21
C:\GGUMLUSE\FT17M8B
1996
21
0
0
5
$\mathrm{~N}$
C:IGGUMLUSE\OUTPUT
C:IGGUMLUSE\TARGI
C:IGGUMLUSE\TARGP
C:IGGUMLUSE\TRANI
C:IGGUMLUSE\TRANP
1

GGUM2000 model 8 was used to calibrate parameters item parameter file from 1 st calibration number of items in 1st calibration person parameter file from 1 st calibration number of respondents in 1 st calibration item parameter file from 2 nd calibration number of items in 2nd calibration person parameter file from 2 nd calibration number of respondents in 2 nd calibration number of anchor items across forms take items from 1 st calibration in sequence take items from 2 nd calibration in sequence number of response categories for all anchor items user will not supply start values for ICC method specify program output file and location target item parameter file on output target person parameter file on output transformed item parameter file on output transformed person parameter file on output ICC method is used to transform model parameters

\section{Example Output File}

The sample output that follows consists of six basic sections, each of which is separated by a section title that is surrounded by asterisks. The first section, entitled "GGUMLINK Version 1," gives information about the current version of the software and how it can be acquired.

The second section of output, entitled "Previous GGUM Calibration," simply identifies the GGUM model that was previously used to derive the parameter estimates in the two groups. Model identification numbers correspond to those used in GGUM2000.

The third section of output, entitled "ICC Method Details," provides information about the iterative algorithm used to produce estimates of linking constants, using the ICC method. This information includes the starting values of the $A$ and $B$ parameters and the value of the criterion function (Equation 17) derived with those starting values. The iterative procedure attempts to find new values for $A$ and $B$ that minimize the criterion function. The number of iterations required to minimize the criterion function and its final value are given, along with the derivatives of the criterion function with respect to $A$ and $B$. The value of the final criterion function should naturally be smaller than its initial value, and its derivatives with respect to both $A$ and B should be very close to zero. In very rare instances, the algorithm may not converge, and the program will run indefinitely. The user can interrupt the program by pressing Cntl-Break and then rerun the program using alternative starting values for $A$ and $B$.

The fourth section of output, entitled "Estimates of Linking Constants," provides the estimates of $A$ and $B$ derived from each of the estimation methods described previously in this article. In cases in which the GGUM 


\section{APPENDIX (Continued)}

parameters are estimated from large samples and contain little estimation error, all of the methods of estimating linking constants will generally yield similar results. This is the case in the present example.

The fifth section of output, entitled "Transformation Information," indicates which linking constant estimates were used to transform GGUM parameter estimates. The metric of parameter estimates from the first group serves as the target metric. The parameters from the second group are transformed to match the target metric. The target parameter estimates and the transformed parameter estimates are printed in two tables, along with their corresponding standard errors. The first table lists the target and transformed $\delta_{i}$ and $\alpha_{i}$ parameters for each item, whereas the second table lists the target and transformed $\tau_{i k}$ parameters for each item.

The sixth and final section of output, entitled "Summary Statistics," lists descriptive statistics for the $\delta_{i}, \alpha_{i}$, and $\theta_{j}$ parameters. Statistics include the number, mean, and standard deviation of each type of parameter estimate. Separate entries are provided for the target parameters and the transformed parameters. Summary statistics are also provided for the original parameters from the second calibration prior to their transformation. These are referred to as initial parameter estimates. The example output follows.

TODAY'S DATE AND TIME:

$01 / 31 / 2003$

$12: 17: 06: 44$

***** GGUMLINK Version $1 * * * * *$

LAST UPDATED ON NOVEMBER 12, 2002

PROGRAMMERS: JAMES S. ROBERTS

CHUN-WEI HUANG

\section{ALL RIGHTS RESERVED, 2002}

THIS PROGRAM HAS BEEN TESTED IN A WINDOWS 98 ENVIRONMENT AND APPEARS TO FUNCTION PROPERLY. HOWEVER, THE AUTHORS ASSUME NO RESPONSIBILITY OR LIABILITY FOR ERRORS THAT MAY OCCUR. PLEASE REPORT ANY PERCEIVED ERRORS TO THE FIRST AUTHOR VIA E-MAIL (JR245@UMAIL.UMD.EDU).

DEVELOPMENT OF GGUMLINK SOFTWARE HAS BEEN PARTIALLY SUPPORTED BY A GRANT FROM THE NATIONAL SCIENCE FOUNDATION UNDER GRANT NO. 0133019, AWARDED BY THE METHODOLOGY, MEASUREMENT, AND STATISTICS PROGRAM (MMS) IN THE DIVISION OF SOCIAL AND ECONOMIC SCIENCES (SES). ANY OPINIONS, FINDINGS, CONCLUSIONS OR RECOMMENDATIONS EXPRESSED IN THIS MATERIAL ARE THOSE OF THE AUTHORS AND DO NOT NECESSARILY REFLECT THE VIEWS OF THE NATIONAL SCIENCE FOUNDATION.

WHEN REPORTING RESULTS DERIVED FROM THIS PROGRAM, PLEASE CITE THE FOLLOWING MANUSCRIPT:

Roberts, J.S. (2001, April). Equating parameters of the generalized graded unfolding model. Paper presented at the annual meeting of the American Educational Research Association, Seattle, WA.

THIS MANUSCRIPT IS AVAILABLE FROM THE ERIC CLEARINGHOUSE ON ASSESSMENT AND EVALUATION.

TO INSURE DISTRIBUTION OF THE MOST CURRENT VERSION OF THIS SOFTWARE, PLEASE DO NOT SHARE COPIES WITH OTHER INDIVIDUALS. INSTEAD, PLEASE ASK OTHERS TO DOWNLOAD THEIR OWN COPIES FROM THE WEB SITE MAINTAINED BY THE FIRST AUTHOR AT:

http://www.education.umd.edu/EDMS/tutorials

THANKS! 
APPENDIX (Continued)

\footnotetext{
***** PREVIOUS GGUM CALIBRATION $* * * * *$

GGUM MODEL 8 USED FOR PARAMETER ESTIMATES

***** ICC METHOD DETAILS $* * * * *$

INITIAL CRITERION FUNCTION $=24.7628654505170$

INITIAL $\mathrm{A}=1.10221556837863$

INITIAL B $=-0.150580226726763$

FINAL SOLUTION REACHED IN 4 ITERATIONS

FINAL SOLUTION CRITERION FUNCTION = 19.6326390865273

DERIVATIVE OF CRITERION WITH RESPECT TO A =

$-3.791786405693554 \mathrm{E}-009$

DERIVATIVE OF CRITERION WITH RESPECT TO B =

1.970380178462072E-009

$* * * * *$ ESTIMATES OF LINKING CONSTANTS $* * * * *$

ICC METHOD A $=1.09507853498482$

ICC METHOD B $=-0.147170026591817$

MEAN-SIGMA METHOD BASED ON ITEM LOCATIONS A $=1.10221556837863$

MEAN-SIGMA METHOD BASED ON ITEM LOCATIONS B $=-0.150580226726763$

MEAN-SIGMA METHOD BASED ON STEP LOCATIONS A $=1.10453136062601$

MEAN-SIGMA METHOD BASED ON STEP LOCATIONS B $=-0.147069494556508$

MEAN-MEAN METHOD BASED ON ITEM LOCATIONS A $=1.08798683792191$

MEAN-MEAN METHOD BASED ON ITEM LOCATIONS B $=-0.148674755817841$

MEAN-MEAN METHOD BASED ON STEP LOCATIONS A $=1.08798683792191$

MEAN-MEAN METHOD BASED ON STEP LOCATIONS B $=-0.158146733908039$

$* * * * *$ TRANSFORMATION INFORMATION *****

TRANSFORMATIONS WILL BE PRODUCED USING RESULTS FROM

THE ICC METHOD.
} 
APPENDIX (Continued)

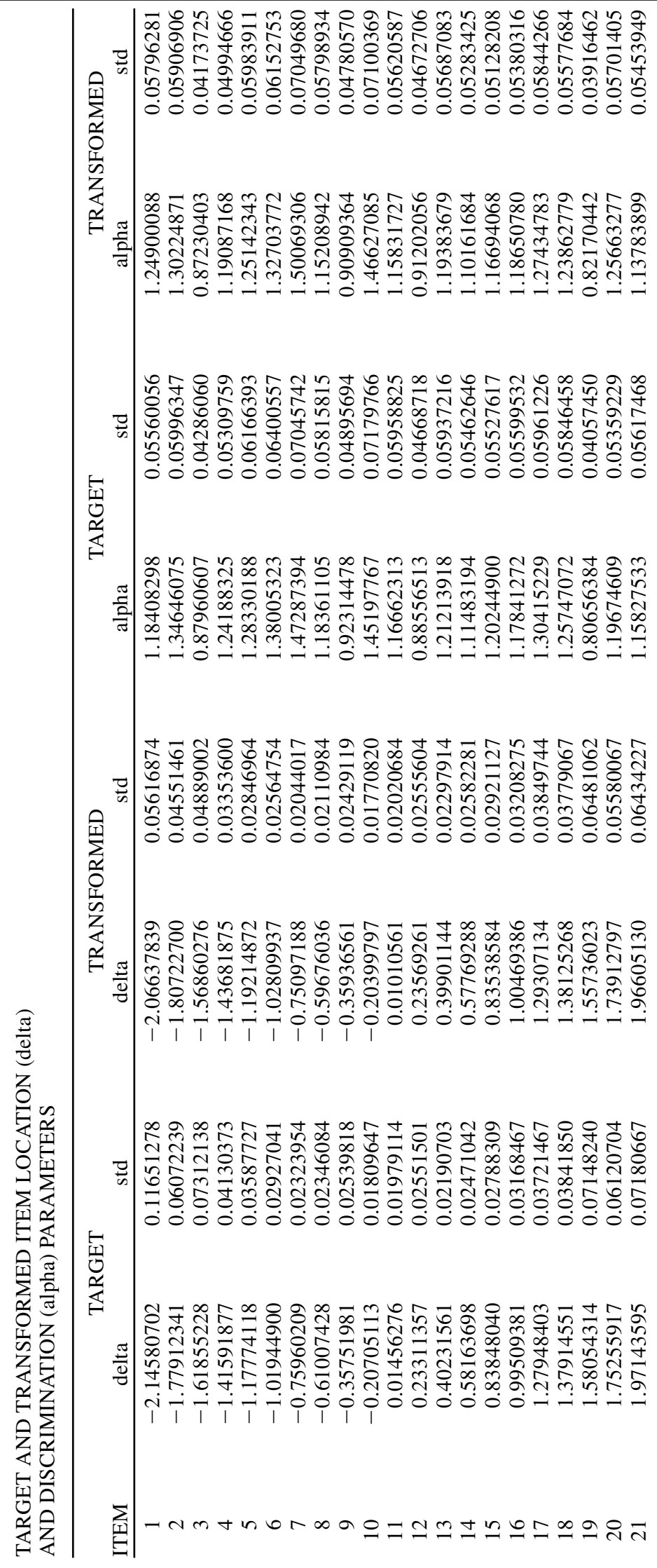


APPENDIX (Continued)

TARGET AND TRANSFORMED THRESHOLD (TAU) PARAMETERS

\begin{tabular}{|c|c|c|c|c|c|}
\hline \multirow[b]{2}{*}{ Item } & \multirow[b]{2}{*}{ Threshold } & \multicolumn{2}{|c|}{ TARGET } & \multicolumn{2}{|c|}{ TRANSFORMED } \\
\hline & & tau & std & tau & std \\
\hline 1 & 0 & 0.00000000 & 0.00000000 & 0.00000000 & 0.00000000 \\
\hline 1 & 1 & -1.90944898 & 0.12621269 & -1.88277507 & 0.07564632 \\
\hline 1 & 2 & -1.45433987 & 0.13363814 & -1.37875935 & 0.08279014 \\
\hline 1 & 3 & -1.09024669 & 0.14413613 & -1.04005716 & 0.08921793 \\
\hline 1 & 4 & -0.56679110 & 0.13852661 & -0.42079407 & 0.08518481 \\
\hline 2 & 0 & 0.00000000 & 0.00000000 & 0.00000000 & 0.00000000 \\
\hline 2 & 1 & -1.73818181 & 0.07756532 & -1.74787266 & 0.07011645 \\
\hline$\overline{2}$ & 2 & -1.45642926 & 0.08421117 & -1.36669865 & 0.07474399 \\
\hline 2 & 3 & -0.90345506 & 0.08487447 & -1.11907927 & 0.07652622 \\
\hline 2 & 4 & -0.35329335 & 0.08476244 & -0.28166050 & 0.07165271 \\
\hline 3 & 0 & 0.00000000 & 0.00000000 & 0.00000000 & 0.00000000 \\
\hline 3 & 1 & -1.81907360 & 0.10215217 & -1.74785496 & 0.09231107 \\
\hline 3 & 2 & -1.36183138 & 0.11170037 & -1.31355465 & 0.09648899 \\
\hline 3 & 3 & -1.03405859 & 0.11227044 & -1.02391169 & 0.09576314 \\
\hline 3 & 4 & -0.47393053 & 0.10532206 & -0.35988861 & 0.09294146 \\
\hline 4 & 0 & 0.00000000 & 0.00000000 & 0.00000000 & 0.00000000 \\
\hline 4 & 1 & -1.82625612 & 0.06854069 & -1.80498884 & 0.06658387 \\
\hline 4 & 2 & -1.36692508 & 0.06999444 & -1.35360619 & 0.07071631 \\
\hline 4 & 3 & -0.90977810 & 0.07175644 & -1.02350046 & 0.06898207 \\
\hline 4 & 4 & -0.33227372 & 0.07017387 & -0.30762360 & 0.06603168 \\
\hline 5 & 0 & 0.00000000 & 0.00000000 & 0.00000000 & 0.00000000 \\
\hline 5 & 1 & -1.79691783 & 0.06722966 & -1.76947286 & 0.06653729 \\
\hline 5 & 2 & -1.23100202 & 0.06616103 & -1.32513580 & 0.06416929 \\
\hline 5 & 3 & -1.03513838 & 0.06719413 & -0.90984621 & 0.06294868 \\
\hline 5 & 4 & -0.30705319 & 0.06045118 & -0.42200271 & 0.05903232 \\
\hline 6 & 0 & 0.00000000 & 0.00000000 & 0.00000000 & 0.00000000 \\
\hline 6 & 1 & -1.81565587 & 0.06141204 & -1.86136024 & 0.06314439 \\
\hline 6 & 2 & -1.28743897 & 0.06032012 & -1.24345238 & 0.06063226 \\
\hline 6 & 3 & -1.02410806 & 0.05894825 & -1.05264445 & 0.05942168 \\
\hline 6 & 4 & -0.34022544 & 0.05246329 & -0.30621089 & 0.05270497 \\
\hline 7 & 0 & 0.00000000 & 0.00000000 & 0.00000000 & 0.00000000 \\
\hline 7 & 1 & -1.84744583 & 0.06062029 & -1.83172716 & 0.05882747 \\
\hline 7 & 2 & -1.19713300 & 0.05433064 & -1.22453633 & 0.05315854 \\
\hline 7 & 3 & -1.00538817 & 0.05113786 & -0.98638197 & 0.04899313 \\
\hline 7 & 4 & -0.25896629 & 0.04649132 & -0.28834852 & 0.04506703 \\
\hline 8 & 0 & 0.00000000 & 0.00000000 & 0.00000000 & 0.00000000 \\
\hline 8 & 1 & -1.64846122 & 0.07727712 & -1.62209863 & 0.07603114 \\
\hline 8 & 2 & -1.35134370 & 0.06706772 & -1.29298749 & 0.06789266 \\
\hline 8 & 3 & -0.97283369 & 0.05973642 & -1.01121796 & 0.06127597 \\
\hline 8 & 4 & -0.26788304 & 0.05535566 & -0.28010057 & 0.05661529 \\
\hline 9 & 0 & 0.00000000 & 0.00000000 & 0.00000000 & 0.00000000 \\
\hline 9 & 1 & -1.81887168 & 0.09968477 & -1.78288713 & 0.09743012 \\
\hline 9 & 2 & -1.12243623 & 0.08548309 & -1.16706474 & 0.08582598 \\
\hline 9 & 3 & -1.19866807 & 0.07811926 & -1.18818200 & 0.07836418 \\
\hline 9 & 4 & -0.31525660 & 0.06623686 & -0.29436463 & 0.06834877 \\
\hline 10 & 0 & 0.00000000 & 0.00000000 & 0.00000000 & 0.00000000 \\
\hline 10 & 1 & -1.76891084 & 0.07045977 & -1.84646440 & 0.06500659 \\
\hline 10 & 2 & -1.41777089 & 0.05819578 & -1.37695156 & 0.05547887 \\
\hline 10 & 3 & -1.01656599 & 0.04770913 & -1.00095557 & 0.04707746 \\
\hline 10 & 4 & -0.31783465 & 0.04197983 & -0.30659356 & 0.04301025 \\
\hline 11 & 0 & 0.00000000 & 0.00000000 & 0.00000000 & 0.00000000 \\
\hline 11 & 1 & -1.58280867 & 0.08257845 & -1.66832116 & 0.07831145 \\
\hline 11 & 2 & -1.32500657 & 0.07097407 & -1.31408569 & 0.06937837 \\
\hline 11 & 3 & -1.01842444 & 0.05989568 & -0.99507576 & 0.06044095 \\
\hline 11 & 4 & -0.37171918 & 0.05147522 & -0.38145515 & 0.05382702 \\
\hline 12 & 0 & 0.00000000 & 0.00000000 & 0.00000000 & 0.00000000 \\
\hline 12 & 1 & -1.71362842 & 0.10412126 & -1.75690705 & 0.09472732 \\
\hline 12 & 2 & -1.37663694 & 0.08951899 & -1.35033431 & 0.08515324 \\
\hline 12 & 3 & -0.98782556 & 0.07499218 & -0.98250852 & 0.07419172 \\
\hline
\end{tabular}


APPENDIX (Continued)

\begin{tabular}{|c|c|c|c|c|c|}
\hline \multirow[b]{2}{*}{ Item } & \multirow[b]{2}{*}{ Threshold } & \multicolumn{2}{|c|}{ TARGET } & \multicolumn{2}{|c|}{ TRANSFORMED } \\
\hline & & tau & std & tau & std \\
\hline 12 & 4 & -0.22253106 & 0.07003362 & -0.23512326 & 0.07048377 \\
\hline 13 & 0 & 0.00000000 & 0.00000000 & 0.00000000 & 0.00000000 \\
\hline 13 & 1 & -1.81771825 & 0.07869944 & -1.82669580 & 0.07300310 \\
\hline 13 & 2 & -1.30498228 & 0.06595588 & -1.27282599 & 0.06614774 \\
\hline 13 & 3 & -0.97682042 & 0.05761176 & -0.99411819 & 0.06033423 \\
\hline 13 & 4 & -0.33284881 & 0.05119242 & -0.32823875 & 0.05439910 \\
\hline 14 & 0 & 0.00000000 & 0.00000000 & 0.00000000 & 0.00000000 \\
\hline 14 & 1 & -1.62556098 & 0.08102722 & -1.72154564 & 0.07638540 \\
\hline 14 & 2 & -1.48633563 & 0.07286680 & -1.40519468 & 0.07226491 \\
\hline 14 & 3 & -0.82233953 & 0.06169342 & -0.87677938 & 0.06396729 \\
\hline 14 & 4 & -0.32830887 & 0.05795025 & -0.30910399 & 0.06131303 \\
\hline 15 & 0 & 0.00000000 & 0.00000000 & 0.00000000 & 0.00000000 \\
\hline 15 & 1 & -1.84536998 & 0.07284492 & -1.79906613 & 0.07166625 \\
\hline 15 & 2 & -1.34027777 & 0.06801038 & -1.28859236 & 0.07110532 \\
\hline 15 & 3 & -1.01774329 & 0.06125107 & -1.12190894 & 0.06592955 \\
\hline 15 & 4 & -0.31679057 & 0.05618296 & -0.26513556 & 0.06053659 \\
\hline 16 & 0 & 0.00000000 & 0.00000000 & 0.00000000 & 0.00000000 \\
\hline 16 & 1 & -1.83765421 & 0.07073781 & -1.82537973 & 0.06849935 \\
\hline 16 & 2 & -1.20213375 & 0.06994910 & -1.23197625 & 0.07049749 \\
\hline 16 & 3 & -1.04885016 & 0.06663573 & -1.08053539 & 0.06786734 \\
\hline 16 & 4 & -0.20544721 & 0.06123847 & -0.17154436 & 0.06438176 \\
\hline 17 & 0 & 0.00000000 & 0.00000000 & 0.00000000 & 0.00000000 \\
\hline 17 & 1 & -1.92916902 & 0.06573504 & -1.88235086 & 0.06643540 \\
\hline 17 & 2 & -1.39028449 & 0.06846175 & -1.40989862 & 0.07135617 \\
\hline 17 & 3 & -0.95900747 & 0.06449757 & -1.02063521 & 0.06760259 \\
\hline 17 & 4 & -0.35322766 & 0.06067613 & -0.33341820 & 0.06515209 \\
\hline 18 & 0 & 0.00000000 & 0.00000000 & 0.00000000 & 0.00000000 \\
\hline 18 & 1 & -1.68423467 & 0.06506445 & -1.68377920 & 0.06538511 \\
\hline 18 & 2 & -1.21632772 & 0.07049047 & -1.23281133 & 0.07150449 \\
\hline 18 & 3 & -0.99404244 & 0.07012623 & -0.95960697 & 0.07334868 \\
\hline 18 & 4 & -0.25812084 & 0.06757342 & -0.28508113 & 0.07172654 \\
\hline 19 & 0 & 0.00000000 & 0.00000000 & 0.00000000 & 0.00000000 \\
\hline 19 & 1 & -1.86930583 & 0.10321562 & -1.74858402 & 0.09717130 \\
\hline 19 & 2 & -1.22060487 & 0.11186470 & -1.27357541 & 0.10878313 \\
\hline 19 & 3 & -1.03290336 & 0.11648712 & -1.02342164 & 0.11392859 \\
\hline 19 & 4 & -0.24170866 & 0.11306690 & -0.19849506 & 0.11408617 \\
\hline 20 & 0 & 0.00000000 & 0.00000000 & 0.00000000 & 0.00000000 \\
\hline 20 & 1 & -1.76077624 & 0.07830272 & -1.80739072 & 0.07449575 \\
\hline 20 & 2 & -1.31188356 & 0.08695802 & -1.27259845 & 0.08123093 \\
\hline 20 & 3 & -0.87784912 & 0.09060174 & -0.85933082 & 0.08793742 \\
\hline 20 & 4 & -0.27228991 & 0.09196842 & -0.30611557 & 0.08980322 \\
\hline 21 & 0 & 0.00000000 & 0.00000000 & 0.00000000 & 0.00000000 \\
\hline 21 & 1 & -1.81693639 & 0.08503926 & -1.78212796 & 0.08263621 \\
\hline 21 & 2 & -1.27064330 & 0.09566960 & -1.27165692 & 0.09203726 \\
\hline 21 & 3 & -0.87078164 & 0.10662159 & -0.87641390 & 0.10688045 \\
\hline 21 & 4 & -0.38304399 & 0.10638751 & -0.39088913 & 0.10710997 \\
\hline
\end{tabular}

\begin{tabular}{llrrr}
\hline GROUP & PARAMETER & $\mathrm{N}$ & \multicolumn{1}{c}{ MEAN } & STD \\
\hline TARGET & DELTA & 21 & -0.0030 & 1.2487 \\
TRANSFORMED & DELTA & 21 & -0.0005 & 1.2406 \\
INITIAL & DELTA & 21 & 0.1339 & 1.1329 \\
TARGET & ALPHA & 21 & 1.1824 & 0.1806 \\
TRANSFORMED & ALPHA & 21 & 1.1747 & 0.1770 \\
INITIAL & ALPHA & 21 & 1.2864 & 0.1939 \\
TARGET & THETA & 1998 & 0.0050 & 0.9855 \\
TRANSFORMED & THETA & 1996 & -0.1421 & 1.0829 \\
INITIAL & THETA & 1996 & 0.0047 & 0.9889 \\
\hline
\end{tabular}

(Manuscript received August 6, 2002;

revision accepted for publication April 10, 2003.) 